\title{
On the terms of geomagnetic daily variation in Antarctica
}

\author{
P. De Michelis, R. Tozzi, and A. Meloni \\ Istituto Nazionale di Geofisica e Vulcanologia, Roma, Italy \\ Received: 5 November 2008 - Revised: 14 May 2009 - Accepted: 1 June 2009 - Published: 22 June 2009
}

\begin{abstract}
The target of this work is to investigate the nature of magnetic perturbations produced by ionospheric and magnetospheric currents as recorded at high-latitude geomagnetic stations. In particular, we investigate the effects of these currents on geomagnetic data recorded in Antarctica. To this purpose we apply a mathematical method, known as Natural Orthogonal Composition, to analyze the magnetic field disturbances along the three geomagnetic field components $(X, Y$ and $Z$ ) recorded at Mario Zucchelli Station (IAGA code TNB; geographic coordinates: $74.7^{\circ} \mathrm{S}, 164.1^{\circ} \mathrm{E}$ ) from 1995 to 1998 . Using this type of analysis, we characterize the dominant modes of the geomagnetic field daily variability through a set of empirical orthogonal functions (EOFs). While such mathematically independent EOFs do not necessarily represent physically independent modes of variability, we find that some of them are actually related to well known current patterns located at high latitudes.
\end{abstract}

Keywords. Geomagnetism and paleomagnetism (Time variations, diurnal to secular) - Magnetospheric physics (Polar cap phenomena; Solar wind-magnetosphere interactions)

\section{Introduction}

Continuous recordings of the elements of the Earth's magnetic field at ground-based observatories show that these elements rarely remain constant for more than an hour or two and if it happens it is generally during the night. In fact, magnetograms are usually characterized by random fluctuations superimposed on a regular trend that is a function of local time, i.e. the solar quiet daily variation $S_{q}$.

Unfortunately, since the daily record of geomagnetic variations results from the overlapping of magnetic fields pro-

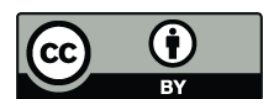

Correspondence to: P. De Michelis (demichelis@ingv.it) duced by different sources, external as well as internal to the Earth, it is extremely difficult to sort out the single contributions. Here, we are interested mainly in those electric current systems, existing in the polar regions, that generate the daily variation.

Indeed, the study of the daily variation above $60^{\circ}$ magnetic latitude is of great importance in understanding the polar cap current systems and the role that these current systems play in the magnetosphere-ionosphere electrodynamic coupling at high latitudes being these current systems mainly driven by particle precipitation and field-aligned currents and depending their configuration on solar wind parameters, magnetic activity and ionospheric electrical conductivity.

In the past, Hasegawa (1939) was the first to find a significant difference between the $S_{q}$ variations in the polar region and those which were expected to arise from the upper atmosphere dynamo process. Later, analyzing geomagnetic data in both Arctic and Antarctic regions, Nagata and Kokubun (1962) found that the geomagnetic daily variation field in the polar cap on geomagnetically quiet days consisted not only of the well established $S_{q}^{0}$ field, which is a smooth extrapolation to the polar region of $S_{q}$ defined at mid and low latitudes, but also of an additional field, the $S_{q}^{p}$ field. In detail, they showed that the associated $S_{q}^{p}$ equivalent current consisted of two parts. One was the dynamo induced current across the pole, approximately from dawn to dusk. The other consisted of two current cells, a counterclockwise cell in the afternoon-evening sector and a clockwise cell in the forenoon-morning sector. Since then the $S_{q}^{p}$ variation has been studied extensively by a number of scientists who attempted to find a three-dimensional current system for the $S_{q}^{p}$ variation.

At present, it is known that the $S_{q}^{p}$ field is mainly generated by a current system limited to latitudes above $60^{\circ}$ and consists of two current vortices confined to the polar cap, with their foci located near the dawn and dusk meridians. However, the extension and the intensity of this current system

Published by Copernicus Publications on behalf of the European Geosciences Union. 
depend on the season. Indeed, in summer the evening cell is much wider and stronger (up to three times) than the morning one, while in winter the two cells are more similar. It is believed that the $S_{q}^{p}$ current system, contrary to the $S_{q}^{0}$ system, is not driven by a ionospheric dynamo due to solar photoionization and tides but rather by external sources (essentially located in the magnetosphere). The pattern of this current system is partially modified by the processes that happen in the magnetosphere and by its interaction with the interplanetary magnetic field (IMF). Indeed, the currents flowing in and near the polar cusp and in the dayside polar cap are coupled, through the field-aligned currents, with the magnetospheric currents. It is for this reason that the $S_{q}^{p}$ current system is strongly enhanced and modified during geomagnetic disturbances and auroral substorms when the effects of nonvanishing transverse IMF components are superimposed to the quiet time configuration of the current system. However, the fact that a twin vortex system appears to be present above $70^{\circ}$ of latitude for all orientations of the IMF, indicates that some elements of the current pattern may be independent of the IMF (Friis-Christensen et al., 1985) and it is this portion of the current system that is the real quiet part of the polar equivalent current system. In the polar regions, however, the quiet-time magnetosphere-ionosphere condition occurs only when the energy transfer from the solar wind to the magnetosphere is at a minimum and even during such quiet conditions, the geomagnetic field at high latitudes is continuously disturbed. Consequently, in these regions quiet days are quite rare events.

Recently, Xu and Kamide (2004) and Chen et al. (2007) have applied a technique, the Natural Orthogonal Component (NOC) analysis, to separate and recognize the different contributions of the ionospheric-magnetospheric current systems to the daily changes of the geomagnetic field as recorded at mid-latitude ground-based geomagnetic observatories. Using this method of analysis, the authors have been able to separate the solar quiet daily variation along the $H$ component from other complicated disturbances. We have extended these works at high latitudes analyzing the hourly values of the three magnetic field components $(X, Y$ and $Z$ ) recorded at the Italian geomagnetic observatory TNB located in Antarctica during a period of 4 years (1995-1998). Indeed, the diurnal variation of the geomagnetic field elements is still not completely understood at high latitudes where the configuration of the electric current systems responsible of the daily geomagnetic field variation is complex. Using the NOC analysis, the time evolution of the geomagnetic field has been studied and it has been found that the first and second order natural components dominate over higher order components also exhibiting features quite similar to those of $S_{q}^{p}$ current systems. However, the aim of our study has been not only to identify the different current systems that contribute to the daily variation of the Earth's magnetic field but also to study their temporal evolution and find possible correlations with appropriate parameters related to these currents.

\section{Data set and method of analysis}

To study the geomagnetic field daily variation in the Antarctic region we used the hourly mean values of the three magnetic field elements which completely describe the magnetic field vector recorded at Terra Nova Bay in Antarctica from 1995 to 1998 (Villante et al., 1997). The geographic and corrected geomagnetic coordinates of Mario Zucchelli geomagnetic observatory at Terra Nova Bay (IAGA code TNB) are $74.7^{\circ} \mathrm{S}, 164.1^{\circ} \mathrm{E}$ and $77.27^{\circ} \mathrm{S}, 278.6^{\circ} \mathrm{E}$ (IGRF95), respectively, while the geographic local time (LT) and corrected magnetic local time (MLT) respect to universal time (UT) are $\mathrm{LT}=\mathrm{UT}+11$ and $\mathrm{MLT}=\mathrm{UT}-8$ according to NASA service http://omniweb.gsfc.nasa.gov/vitmo/cgm_vitmo.html.

At this geomagnetic observatory, three-axis flux gate magnetometers oriented in the reference system $H D Z$ are installed. Consequently at TNB observatory, the magnetic field is measured in a coordinate system where the $H$ component point towards magnetic north, the $D$ component to magnetic east, and the $Z$ component is orthogonal to both of them, pointing downward. The orientation of this kind of coordinate system therefore depends on the location the magnetic field is being measured at.

In this study we have transformed the geomagnetic field horizontal components ( $H$ and $D$ ) into $X$ and $Y$ components, which are directed along the geographic meridian and parallel, respectively. As angular rotation between the geographic and magnetic meridian, we have considered the average value of the declination angle $D$ at TNB observatory, which is equal to $136^{\circ}$. This transformation is necessary being the reconstruction of the equivalent current systems in the polar region generally obtained from the $X, Y$ and $Z$ magnetic field values. Indeed, it is known that there is a considerable deviation between the direction of the $H$ component (magnetic north) and the $X$ component (geographic north) especially in the higher-latitudes observatories. At high latitudes the magnetic perturbation in the geographic northward component depends almost equally on the magnetic perturbation along $H$ and $D$ and even the sign of the magnetic perturbation along $X$ can be opposite to the sign of the perturbation along $H$. For this reason, to compare our results with those obtained using different methods of analysis, it is important to use the same reference system.

TNB geomagnetic observatory is generally located inside the polar cap quite close to the auroral oval but, under particular geomagnetic conditions and for a particular range of MLTs, it may be placed under the southern polar cups (Pietrolungo et al., 2008) and thus the geomagnetic variations in this condition may be heavily influenced by local cusp phenomena, mainly related to field aligned currents (Matsushita and Xu, 1982; Campbell, 1997). The structure of the geomagnetic daily variation is consequently quite complex in this part of the world and the use of the Natural Orthogonal Component analysis (Jackson et al., 1991; Golovkov et al., 
1992; Xu and Kamide, 2004) can be a tool extremely useful to reveal simple patterns within it.

The Natural Orthogonal Component (NOC) analysis is, indeed, a powerful and elegant method of data analysis aimed at obtaining low-dimensional approximate descriptions of high-dimensional processes. In particular, this method of analysis offers a way to extract those structures that remain coherent throughout a time series. In practice, given a set of observations it is possible to estimate a set of independent eigenvectors and eigenvalues whose combination allows writing the observed variables in terms of a new basis that is just a rotated version of the original observations. This type of analysis has been developed by several authors and widely used in literature, for instance for the study of daily magnetic variation (Golovkov et al., 1978; Xu and Kamide, 2004; Chen et al. 2007), for the study of global models of the geomagnetic field (Xu, 2002, 2003), for the automatic computation of $K$ indices (Golovkov et al., 1989; Papitashvili et al., 1992), and even for the separation of the substorm current system into directly driven and loading-unloading components (Sun et al., 1998, 2000).

Before describing the application of the NOC method to our data set, a brief introduction follows. Let us suppose to have a variable $x\left(d_{i}, t_{j}\right)$ representing the value of a magnetic field element $(X, Y, Z)$ for a certain day $d_{i}$, and time $t_{j}$. Given a number of samples of $x\left(d_{i}, t_{j}\right)$ at different days and times, the application of the NOC method allows extracting a smaller set of variables, let's say of Empirical Orthogonal Functions (EOFs) and Principal Components (PCs), able to describe the whole set of observations. Among the different techniques used for data representation the NOC method has the peculiarity that the set of functions used for the expansion of the time series is estimated using only the observed dataset. This is the main difference with other methods, as for instance the spherical harmonic and Fourier analyses, where the fundamental orthogonal basis set is artificially chosen a priori.

Therefore, we can decompose the daily variation of any geomagnetic component in terms of a basis of Empirical Orthogonal Functions (EOFs) $\phi^{k}\left(t_{j}\right)$ :

$x\left(d_{i}, t_{j}\right)=\sum_{k=1}^{K} w_{k} A^{k}\left(d_{i}\right) \phi^{k}\left(t_{j}\right)$

where the collection of values $x\left(d_{i}, t_{j}\right)$ are the elements $x_{i j}$ of the matrix $\mathbf{X}(m \times n)$ whose rows correspond to the days $(i=1,2, \ldots, m)$ and columns correspond to the time $(j=1$, $2, \ldots, n), K$ is the number of components chosen for the decomposition (i.e. the truncation level), $\phi^{k}\left(t_{j}\right)$ is the mode (EOF) of the $k$-th component describing the daily variation (i.e. is the basis used for the expansion), $A^{k}\left(d_{i}\right)$ is the Principal Component (PC) and represents the amplitude of $k$-th mode, and $w_{k}$ is the associated weigh.

To evaluate the EOFs and the associated PCs from a dataset the error made in the representation of the observed

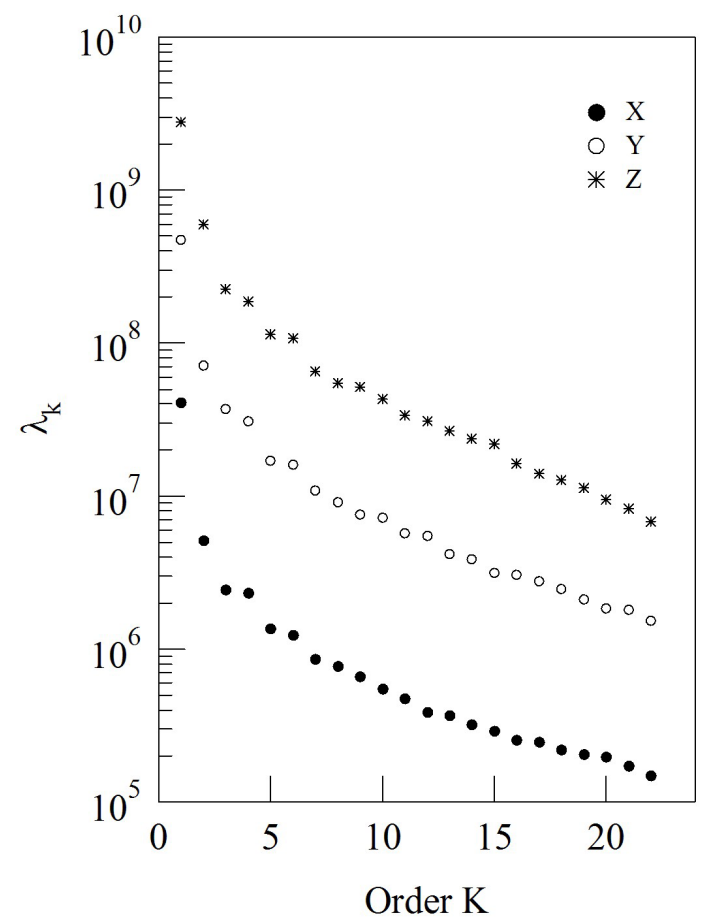

Fig. 1. The NOC eigenvalue spectra $\lambda_{k}$ of $X, Y$ and $Z$ magnetic field components recorded at Mario Zucchelli Station (TNB) in Antarctica. The $\lambda_{k}$ spectra of $Y$ and $Z$ components have been scaled by a factor of 10 and $10^{2}$, respectively, for convenience.

data by means of the expansion (1) is to be minimized. This error depends on the truncation level $K$ : the higher the truncation level, the lower the error.

Since we are looking for a number of components able to describe completely the observed variable, one additional assumption is that the EOFs $\left(\phi^{k}\right)$ are orthogonal and the PCs $\left(A^{k}\right)$ vary independently. This leads to solve the well-known eigenvalue-eigenvector problem that permits an estimation of the eigenvalues $\lambda_{k}\left(\lambda_{k}=1 / w_{k}\right)$ together with the corresponding eigenvectors $\phi^{k}$ and amplitudes $A^{k}$ for $k=1, \ldots, K$ (see for more details Xu and Kamide, 2004).

In what follows we try to find a correspondence between the main EOFs and the real current patterns flowing in the ionosphere and magnetosphere in the polar region. However, it is important to underline that the EOFs and PCs found in this way do not necessarily correspond to real current systems. In fact, while the EOFs are orthogonal by definition, the currents are generally not.

We stress also that NOC technique is strictly valid for signals resulting from the linear superposition of independent modes. Therefore, cases of signals deriving from non linear processes could lead to results difficult to interpret. 

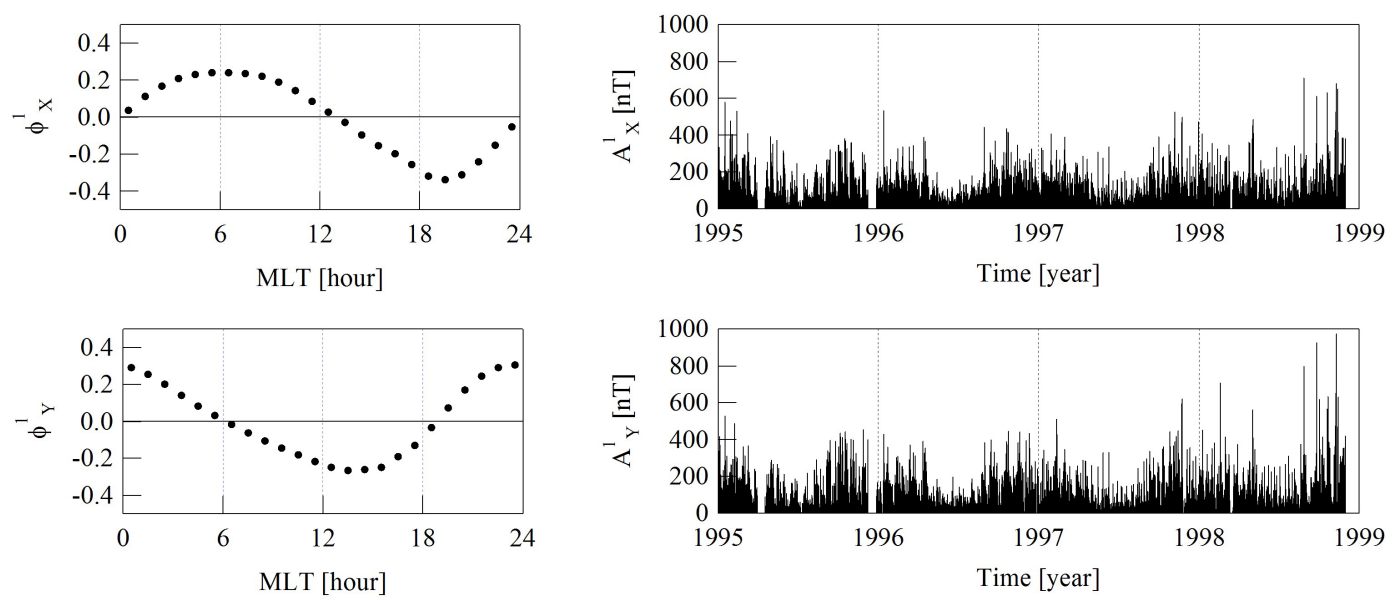

Fig. 2. On the left, the first EOF associated to the $X$ (top) and $Y$ (bottom) components, respectively; on the right, their associated PCs.

\section{Results and discussion}

As already mentioned in the previous section, we used the hourly average values of the variations of the $X, Y$ and $Z$ magnetic field components recorded at TNB geomagnetic observatory from 1 January 1995 to 30 November 1998. On the long term side these data are characterized by annual as well as secular changes. For this reason, before applying the NOC analysis, we have removed the daily mean from each day eliminating, in this way, the trend caused by the secular changes and other possible long term drift.

Figure 1 shows the spectra of the eigenvalues $\lambda_{k}$ obtained applying the NOC analysis to the three magnetic field elements. A rapid drop characterizes all spectra, and the energy is mainly concentrated in the lowest orders. As a matter of fact, the first two eigenvalues (and consequently the two associated PCs) explain up to $77 \%, 76 \%$ and $75 \%$ of the total variance of $X, Y$ and $Z$ magnetic field elements, respectively. This implies that the first few EOFs play a role much more relevant than the others and, consequently, the daily variation of the geomagnetic field at high latitudes can be well reconstructed by considering only these few NOC terms.

In Figs. 2, 3 and 4, we have grouped the EOFs and PCs according to the different current systems we think they may represent. Since we believe that the first $(k=1)$ EOF associated to $X$ and $Y$ elements $\left(\phi_{X}^{1}\right.$ and $\left.\phi_{Y}^{1}\right)$ may represent the $S_{q}^{p}$ current system, we have grouped them and the corresponding PC ( $\phi_{X}^{1}$ and $\left.\phi_{Y}^{1}\right)$ in Fig. 2. Indeed, if we take into account the different directions assumed by the two EOFs simultaneously in the different magnetic local time sectors (00:00-06:00; 06:00-12:00; 12:00-18:00 and 18:0024:00) we can easily reconstruct the pattern of the current system underneath which TNB geomagnetic observatory rotates. The pattern so inferred consists of two currents flowing in opposite directions from 24:00 to 12:00 MLT, one crossing 18:00 MLT and the other 06:00 MLT. This result is well in agreement with the structure of the $S_{q}^{p}$ current system, which is formed by two vortices where the currents flow in opposite directions. Indeed, what we are able to reconstruct from our dataset is only the outer portion of this current system. The PCs $\left(A_{X}^{1}\right.$ and $\left.A_{Y}^{1}\right)$ associated to these EOFs are plotted in the right panels of the same figure. These components display a seasonal trend characterized by a maximum in the austral summer and a minimum in the austral winter. This seasonal trend is in agreement with the characteristic of the $S_{q}^{p}$ field intensity to show a strong seasonal variation. Indeed, while the total current associated to the $S_{q}^{p}$ system amounts to about $15 \times 10^{4} \mathrm{~A}$ in the sunlit polar cap (summer), it is reduced to less than one third of its value when the polar cap becomes dark (winter) (Xu, 1989).

It should be pointed out that, in addition to its seasonal variation, $S_{q}^{p}$ depends on magnetic activity and IMF sector polarity. Indeed, it has been shown that when magnetic activity increases, the $S_{q}^{p}$ current pattern is distorted while the position of the vortices is shifted toward either the morning side or evening side according to sector polarities of IMF (FriisChristensen, 1984). We have analysed the existence of this possible relationship studying the correlation between the PCs $\left(A_{X}^{1}\right.$ and $\left.A_{Y}^{1}\right)$ and some significant parameters: the solar wind dynamic pressure $P_{\mathrm{dyn}}\left(P_{\mathrm{dyn}}=\rho v^{2}\right.$, where $\rho$ is the solar wind mass density and $v$ is its flow speed), the north-south component $\left(B_{z}\right)$, the southward $\left(B_{S}\right)$ and the eastward $\left(B_{y}\right)$ components of the interplanetary magnetic field (IMF) in the GSM system, the so-called energy coupling function $\varepsilon$ (Akasofu, 1978), which is a measure of the energy flux that flows into the magnetosphere, and the $K_{p}$ index, which is an indication of the level of geomagnetic perturbation on planetary scale (data come from OMNI2 database).

To find, where possible, these correlations we have evaluated the Pearson's $|r|$ coefficient. As well known, this coefficient $|r|$ provides a simple measure of the linear correlation ranging from 0 and 1 . A value of 1 is the result of a perfect 

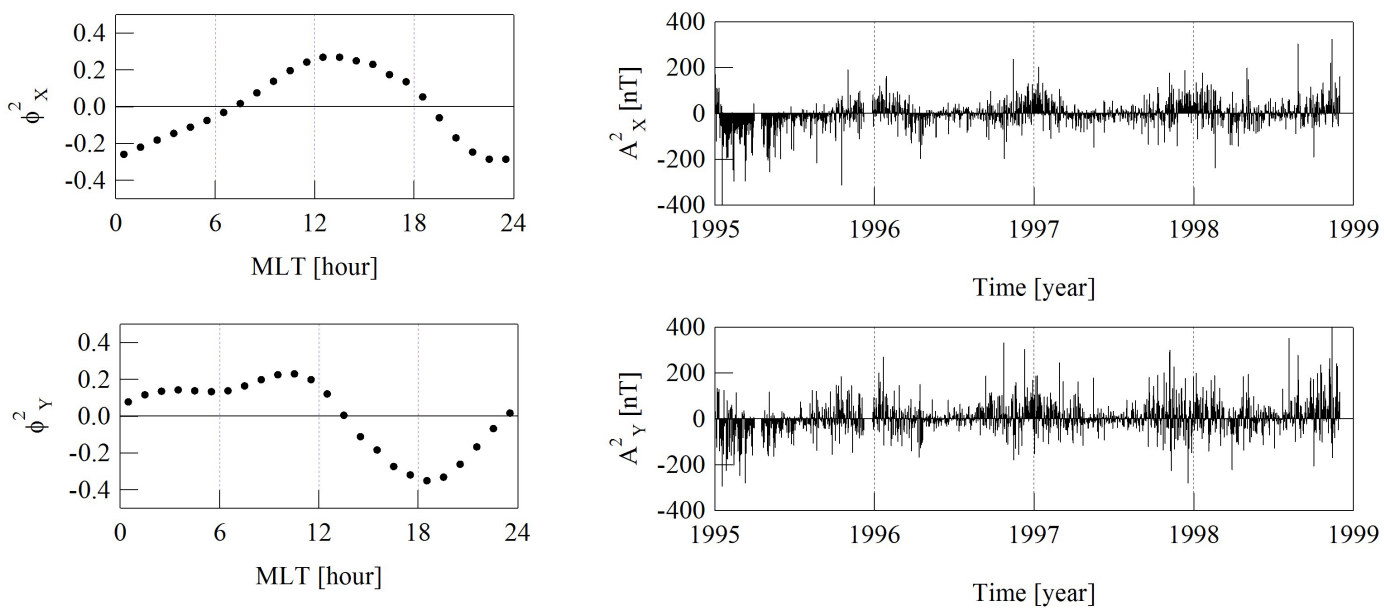

Fig. 3. On the left, the second EOF associated to the $X$ (top) and $Y$ (bottom) components, respectively. On the right, the PCs associated to each EOF.

linear relationship between the analyzed variables, while a value of 0 is the result of no linear relationship. In practice, the value of this parameter is some intermediate number whose significance depends on the number of samples.

In order to establish a significance level for the correlation found, we have fixed a threshold $\left|r_{s}\right|$ for the Pearson's coefficient. This threshold has been evaluated according to the significance level of $1 \%$ for samples characterized by a degree of freedom equal to 1000 . The chosen threshold is $\left|r_{s}\right|=0.081$ (Fisher, 1972). It means that in our case values smaller than 0.081 can be read as absence of correlation.

In Table 1 we have reported the correlation coefficients between the PCs and the selected parameters. It is necessary to notice that the correlation between a PC and one parameter will be found only if this parameter produces a change in the intensity of the current system described by the EOF associated to the PC. Indeed, the PC describes the temporal evolution of a specific spatial mode, i.e. EOF, and it cannot consequently describe any distortion of the current system due to different magnetospheric configuration.

According to the results reported in Table 1, the first PC associated to the $X$ component $\left(A_{X}^{1}\right)$ is very well correlated with $K_{p}, B_{z}, B_{s}, \varepsilon$, and $P_{\text {dyn. }}$. The same results have been obtained correlating the PC associated to the $Y$ component $\left(A_{Y}^{1}\right)$ with the same parameters (data reported in Table 1). These results support the hypothesis that the first $(k=1)$ EOFs associated to $X$ and $Y$ components $\left(\phi_{X}^{1}\right.$ and $\left.\phi_{Y}^{1}\right)$ may represent the $S_{q}^{p}$ current system. Indeed, the selected parameters, well characterizing the impact of the solar wind on the magnetosphere, can affect the $S_{q}^{p}$ current pattern.

It is worth noticing that this result is in agreement with the reconstruction of equivalent ionospheric currents above Antarctica proposed by Papitashvili et al. (1990). Using hourly mean values of geomagnetic field horizontal components $X$ and $Y$ from 20 Antarctic observatories and auto-
Table 1. The Pearson's $|r|$ linear coefficient. The "-_" refers to values less than the fixed threshold value $\left|r_{s}\right|=0.081$.

\begin{tabular}{ccccccc}
\hline & $A_{X}^{1}$ & $A_{X}^{2}$ & $A_{Y}^{1}$ & $A_{Y}^{2}$ & $A_{Z}^{1}$ & $A_{Z}^{2}$ \\
\hline$K_{p}$ & 0.67 & 0.13 & 0.63 & - & 0.41 & 0.22 \\
$P_{\text {dyn }}$ & 0.35 & 0.11 & 0.28 & - & 0.14 & 0.14 \\
$B_{Z}$ & 0.41 & - & 0.38 & - & 0.37 & - \\
$B_{S}$ & 0.53 & 0.10 & 0.46 & 0.13 & 0.45 & - \\
$B_{y}$ & - & 0.15 & 0.12 & 0.25 & - & 0.12 \\
$\varepsilon$ & 0.57 & - & 0.53 & 0.10 & 0.44 & - \\
\hline
\end{tabular}

matic stations, Papitashvili et al. (1990) have recognized the existence of three different types of ionospheric convection patterns: a) a two-vortex system controlled by the "quasiviscous" interaction and southward IMF; b) a zonal current system, controlled by azimuthal IMF; and c) a two-vortex system controlled by the northward IMF.

According to this analysis our first mode, associated to the $X$ and $Y$ components $\left(\phi_{X}^{1}\right.$ and $\left.\phi_{Y}^{1}\right)$, seems to identify the first type of the equivalent current system found by Papitashvili et al. (1990).

The EOFs $\phi_{X}^{2}$ and $\phi_{Y}^{2}$ are reported in Fig. 3. Taking into account the different directions assumed by the two EOFs simultaneously in the different magnetic local time sectors we can reconstruct, also in this case, the pattern of the current system flowing above TNB geomagnetic observatory. The pattern so inferred consists of a current approximately from dawn to dusk. The PCs $\left(A_{X}^{2}\right.$ and $\left.A_{Y}^{2}\right)$ associated to these EOFs are characterized by a seasonal trend with a minimum in the austral winter and a maximum in the austral summer (Fig. 3). We believe that these EOFs may be considered representative of the dawn-to-dusk Pedersen current. 

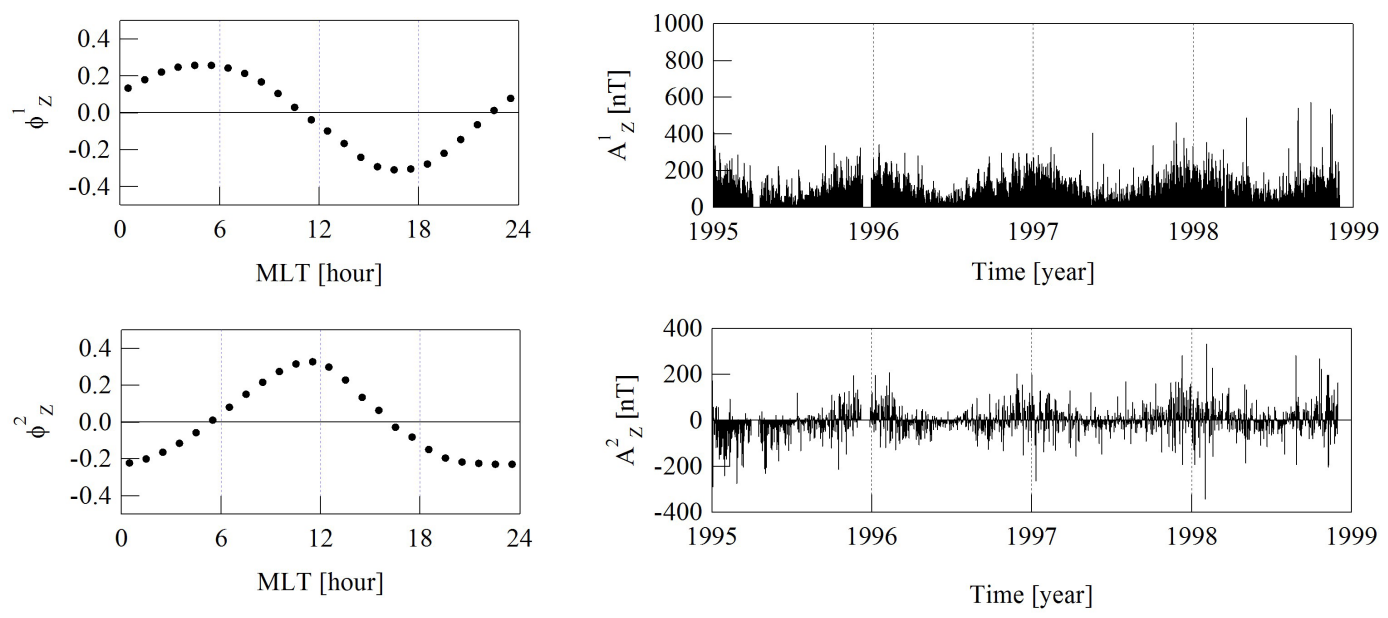

Fig. 4. On the left, the first (top) and the second (bottom) EOF associated to the $Z$ component. On the right, the PCs associated to each EOF.

What is interesting to notice is that the PCs $A_{X}^{2}$ and $A_{Y}^{2}$, associated to the EOFs $\phi_{X}^{2}$ and $\phi_{Y}^{2}$, are weakly correlated with some interplanetary quantities (see Table 1 ) and in particular these PCs are correlated to the daily average direction of the $B_{y}$ component of the IMF. The current system, which is described by the second EOFs associated to the $X$ and $Y$ components of the geomagnetic field, seems to be consequently influenced by the Svalgaard-Mansurov effect (Svalgaard, 1968; Mansurov, 1969). According to this effect, indeed, the ground-based geomagnetic observations and in particular the daily variation of the geomagnetic field at high (cusp and polar cap) latitudes are influenced by the heliospheric magnetic field sector polarity being the sign of the $B_{y}$ responsible for the direction of the east-west flowing ionospheric current. This correlation is not so obvious for a current system that should represent the Pedersen currents. It is known indeed that the pattern of the Pedersen currents does not change its direction and it cannot be consequently influenced by the $B_{y}$ component of the IMF. However, we know that in the polar cap exists a zonal current around the geomagnetic pole (see for example Papitashvili et al., 1990), which flows anticlockwise for $B_{y}>0$ and in the opposite direction for $B_{y}<0$. We can suppose that the EOFs $\phi_{X}^{2}$ and $\phi_{Y}^{2}$, in addition to the Pedersen currents, contain this zonal current system, which the NOC analysis is not able to recognize and separate.

Figure 4 shows the trends of the first $\left(\phi_{Z}^{1}\right)$ and second $\left(\phi_{Z}^{2}\right)$ EOFs associated to the $Z$ component in magnetic local time (MLT). The daily trend of the $\phi_{Z}^{1}$ is characterized by a maximum in the morning sector and a minimum in the afternoon one while the $\phi_{Z}^{2}$ function displays a single maximum at around noon.

The recognition of the current systems associated to these EOFs is not simple. From a theoretical point of view, if we divide the current systems responsible of the geomagnetic variations into toroidal and poloidal systems, we have that the toroidal current flowing in the ionosphere sheet layer will produce poloidal magnetic perturbations while the poloidal one, consisting of field-aligned currents and their closing irrotational currents in the ionospheric current sheet, will produce toroidal magnetic perturbations above the ionosphere and no perturbations below (Richmond, 2002). This means that the ground-based magnetometers should respond almost exclusively to poloidal magnetic fields due to the solenoidal current circuit while they shouldn't measure those toroidal fields due to the poloidal current circuit.

At high latitudes as poloidal current circuits we have mainly the Hall and Pedersen currents. The Pedersen current is perpendicular to the $Z$ axis and parallel to the electric field created by the field-aligned currents, while the Hall current is perpendicular to both the $Z$ axis and the electric field (Tascione, 1988).

However, analysing the effect of these current systems on the $Z$ component of the geomagnetic field it is important to say that according to the Fukushima theorem (Fukushima, 1976), under the assumption of field-aligned currents (FAC) parallel to the $Z$ axis and a ionosphere with uniform conductivity parallel to the ground, the field-aligned and Pedersen currents combined will produce no ground magnetic signature. However, if the inclination of the ambient field deviates slightly from vertical (e.g. inclination $\approx 80^{\circ}$ at auroral latitudes) some magnetic effects from the FAC-Pedersen current circuit will leak to the ground and we will find a small fieldaligned contribution from this circuit.

We believe that the first natural component associated to the $Z$ component $\left(\phi_{Z}^{1}\right)$, as well as those associated to the other two magnetic field components $X$ and $Y$, may describe the effect on the ground produced by two vortices with their foci located near dawn and dusk meridians, which is well known to be associated with global plasma convection in the magnetosphere. This current system is characterized by an intensity that is stronger in summer than in winter, as 
described by the temporal trend of the PC $\left(A_{Z}^{1}\right)$ associated to this $\operatorname{EOF}\left(\phi_{Z}^{1}\right)$, and it is correlated to the $\varepsilon$ coupling function, the southward $\left(B_{s}\right)$, the north-south $\left(B_{z}\right)$ component of the interplanetary magnetic field, the dynamic pressure $P_{\text {dyn }}$ and the $K_{p}$ index (see Table 1).

The second natural component $\phi_{Z}^{2}$, which is characterized by positive values in the time interval 06:00 $<$ MLT $<16: 00$, could be the effect on the ground of the Pedersen currents. Indeed, what is important to notice is that the eigenvalue associated to the second natural component is, in first approximation, one order less than that associated to the first natural component. So, as expected, the perturbation on the ground produced by the Pedersen currents along the $Z$ magnetic field component is small.

\section{Summary and conclusions}

Ground magnetic perturbations have been widely used to study magnetospheric and ionospheric dynamics, since the magnetic data obtained at the Earth's surface include valuable information about a variety of current sources flowing in the ionosphere, magnetosphere, at the magnetopause, and within the Earth's interior. However, one of the major problems it has always been to evaluate the relative importance of these currents in generating particular patterns or modes of global or local magnetic perturbations under study. We have tried to overcome this problem using the NOC analysis. Investigating the nature of the daily magnetic variations recorded at an Antarctic ground observatory, we have found that for $X, Y$ and $Z$ magnetic field components the main contribution to the polar daily variation comes from the Hall currents. This current system, which is a permanent feature of the polar region, largely depends upon solar wind parameters and magnetospheric conditions. Indeed, we have found that the PCs $\left(A_{X}^{1}, A_{Y}^{1}\right.$ and $\left.A_{z}^{1}\right)$, associated to those EOFs $\left(\phi_{X}^{1}, \phi_{Y}^{1}\right.$ and $\phi_{Z}^{1}$ ) describing the Hall current system, exhibit, as expected, a correlation with the solar wind dynamic pressure $\left(P_{\text {dyn }}\right)$, the $B_{z}$ and $B_{s}$ interplanetary magnetic field components, the energy coupling function $\varepsilon$ and the $K_{p}$ index. The second important contribution to the polar daily variation of the geomagnetic field mainly comes from Pedersen currents. However we think that this second mode, obtained from the NOC analysis, may contain also a contribution coming from a zonal current system present at high latitude and controlled by the azimuthal IMF (Papitashvili et al., 1990).

These results, which are well in agreement with the current systems present at high latitude, show some differences with respect to the results recently presented by Pietrolungo et al. (2008). Indeed, the authors, using data coming from three different geomagnetic observatories all located within the Antarctic polar cap, have suggested that the contribution of the $S_{q}^{p}$ polar electric current systems to the diurnal variation is not relevant and that the mid latitude ionospheric cur- rents are the main source of the daily variation also at high latitudes, at least during a period of solar minimum.

On the contrary, according to our analysis the dominant contribution is given by the Hall and Pedersen currents and being this result obtained for a period around a minimum of solar activity (the lowest number of sunspot cycle 22 was officially recorded in May 1996) it is reasonable to extend it also to the years characterized by a mid and high solar activity.

The difference between our results and those proposed by Pietrolungo et al. (2008) is due to the different approach used in the data analysis. The superposed epoch analysis, which has been applied by Pietrolungo et al. (2008) to reconstruct the average diurnal variation along $X$ and $Y$ components of the geomagnetic field, does not allow recognizing the different contributions of the ionospheric-magnetospheric current systems to the daily changes of the geomagnetic field. This method, indeed, gives only an average trend of daily variation where the different contributions are contained all together. The results reported in their analysis correspond to our first mode, which representing the dominant mode associated to the highest energetic eigenvalue, well describe the average patterns. Indeed, taking into account the relationship between LT and MLT at TNB geomagnetic observatory (LT=MLT+19), the $\phi_{X}^{1}$ is characterized by a minimum around 14:00 LT while the $\phi_{Y}^{1}$ function is characterized by negative values in the range 00:00 $<\mathrm{LT}<14: 00$ with a minimum around $\mathrm{LT}=09: 00$ and by positive values in the range 14:00 $<\mathrm{LT}<24: 00$ with a maximum around $\mathrm{LT}=18: 30$. Our patterns are consequently equal to those reported by Pietrolungo et al. (2008). These patterns of the $X$ and $Y$ components might resemble the $S_{q}^{0}$ current system at high latitude in the Southern Hemisphere. Nevertheless, the daily pattern of the $\phi_{Z}^{1}$ in LT is opposite to what is expected, i.e. it is characterized by minimum around noon instead of a maximum. So, the simultaneous analysis of the three magnetic field components in magnetic local time excludes the possibility that the daily magnetic variation results from the $S_{q}^{0}$ current system and confirms the importance of the Hall currents in the polar region.

Acknowledgements. The research activity at TNB is supported by Italian PNRA (Programma Nazionale di Ricerche in Antartide). OMNI2 database is provided by the National Space Science Data Center.

Topical Editor I. A. Daglis thanks M. Freeman and another anonymous referee for their help in evaluating this paper.

\section{References}

Akasofu, S. I.: Interplanetary energy flux associated with magnetospheric substorms, Planet.Space Sci., 27, 425-431, 1978.

Campbell, W. H.: Introduction to geomagnetic fields, Cambridge University Press, 135-142, 1997.

Chen, G.-X., Xu, W. Y., Du, A. M., Wu, Y. Y., Chen, B., and Liu, X. C.: Statistical characteristics of the day-to-day variabil- 
ity in the geomagnetic Sq field, J. Geophys. Res., 112, A06320, doi:10.1029/2006JA012059, 2007.

Fisher, R. A.: Statistical methods for research workers, 14th ed., Hafner Press, 1972.

Friis-Christensen, E.: Polar cap current systems, In Magnetospheric currents, edited by: Potemra, T. A., AGU, Washington D.C., pp. 86-95, 1984.

Friis-Christensen, E., Kamide, Y., Richmond, A. D., and Matsushita, S.: Interplanetary magnetic field control of high-latitude electric field and currents determined from Greenland magnetometer data, J. Geophys. Res., 90, 1325-1338, 1985.

Fukushima, N.: Generalized theorem for no ground magnetic effect of vertical currents connected with Pedersen currents in the uniform-conductivity ionosphere, Rep. Ionos. Space Res. Jap., 30, 35-40, 1976.

Golovkov, V. P., Papitashvili, N. E., Tyupkin, Y. S., and Kharin, E. P.: Separation of geomagnetic field variations on the quiet and disturbed components by the MNOC, Geomagnetism and Aeronomy, 18, 511-515, 1978.

Golovkov, V. P., Papitashvili, V. O., and Papitashvili, N. E.: Automated calculation of the $\mathrm{K}$ indices using the method of natural orthogonal components, Geomagnetism and Aeronomy, 29, 514-517, 1989.

Golovkov, V. P., Kozhoyeva, K. G., and Shkolnikova, S. I.: The use of the method of natural orthogonal components for separation of partially nonorthogonal variations of the geomagnetic field, Geomagnetism and Aeronomy, 32, 715-717, 1992.

Hasegawa, M.: Provisional report of the statistical study on the diurnal variations of terrestrial magnetism in the north pole regions, Trans. Washington Meeting, IUGG-ATME, Bull. No. 11 pp. 311-318, A.H.R Gddie, ed. Edinburg, 4-15 September 1939.

Jackson, G. M., Mason, I. M., and Greenhalgh, S. A.: Principal component transforms of triaxial recordings by singular value decomposition, Geophysics, 56, 528-533, 1991.

Mansurov, S. M.: New evidence of a relationship between magnetic field in space and on earth, Geomagnetism and Aeronomy, 9, 622-623, 1969.

Matsushita, S. and Xu, W.: Equivalent ionospheric current system representing solar daily variations of the polar geomagnetic field, J. Geophys. Res., 87, 8241-8254, 1982.

Nagata, T. and Kokubun, S.: A particular geomagnetic daily variation $S_{q}^{p}$ in the polar regions on geomagnetically quiet days, Nature, 195, 555-557, 1962.
Papitashvili, V. O., Feldstein, Y. I., Levitin, A. E., Belov, B. A., Gromova, L. I., and Valchuk, T. E.: Equivalent ionospheric currents above Antactica during the austral summer, Antarctic Science, 2(3), 267-276, 1990.

Papitashvili, N. E., Papitashvili, V. O., Belov, B. A., Hakkinen, L., and Sucksdorff, C.: Magnetospheric contribution to K-indices, Geophys. J. Int., 111, 348-356, 1992.

Pietrolungo, M., Lepidi, S., Cafarella, L., Santarelli, L., and Di Mauro, D.: Daily variation at three Antarctic geomagnetic observatories within the polar cap, Ann. Geophys., 26, 2179-2190, 2008, http://www.ann-geophys.net/26/2179/2008/.

Richmond, A. D.: Modeling the geomagnetic perturbations produced by ionospheric currents, above and below the ionosphere, Journal of Geodynamics, 33, 143-156, 2002.

Tascione, T. F.: Introduction to space environment, Orbit Book Company, Malablar, Flor., 1998.

Svalgaard, L.: Sector structure of the interplanetary magnetic field and daily variation of the geomagneti field at high latitudes, Geophysical Papers R-6, Danish Meteorological Institute, Charlottenlund, 1968.

Sun, W., Xu, W.-Y., and Akasofu, S.-I.: Mathematical separation of directly driven and unloading components in the ionospheric equivalent currents during substorms, J. Geophys. Res., 103, 11695-11700, 1998.

Sun, W., Xu, W.-Y., and Akasofu, S.-I.: An improved method to deduce the unloading component for the magnetospheric substorms, J. Geophys. Res., 105, 13131-13140, 2000.

Villante, U., Lepidi, S., Francia, P., Meloni, A., and Palangio, P.: Long period geomagnetic fluctuations at Terra Nova Bay (Antarctica), Geophys. Res. Lett., 24, 1443-1446, 1997.

Xu, W.-Y.: Polar region Sq, PAGEOPH, 131, 371-393, 1989.

$\mathrm{Xu}$, W.-Y.: Revision of the high-degree Gauss coefficients in the IGRF 1945-1955 models by using natural orthogonal component analysis, Earth Planets Space, 54, 753-761, 2002.

$\mathrm{Xu}$, W.-Y.: Natural orthogonal component analysis of international geomagnetic reference field models and its application to historical geomagnetic models, Geophys. J. Int., 152, 613, doi:10.1046/j.1365-246X.2003.01875.x, 2003.

$\mathrm{Xu}, \mathrm{W} .-\mathrm{Y}$. and Kamide, Y.: Decomposition of daily geomagnetic variations by using method of natural orthogonal component, J. Geophys. Res., 109, A05218, doi:1029/2003JA010216, 2004. 